\title{
Bovine vitreous gel can reactivate replicative senescence of human dermal fibroblast
}

\author{
Maria Vianney Sansan ${ }^{1}$, Sunardi Radiono ${ }^{1}$, Muhamad Eko Irawanto ${ }^{2}$, and Yohanes Widodo Wirohadidjojo ${ }^{1, *}$ \\ ${ }^{1}$ Department of Dermatology and Venereology, Faculty of Medicine, Universitas Gadjah Mada and Dr. Sardjito General Hospital, \\ Yogyakarta, Indonesia \\ ${ }^{2}$ Department of Dermatology and Venereology, Faculty of Medicine, Sebelas Maret University and Dr. Moewardi General Hospital, \\ Surakarta, Indonesia \\ *Corresponding author: widiokarsono@yahoo.com
}

SUBMITTED 12 September 2017 REVISED 13 Maret 2018 ACCEPTED 23 May 2018

\begin{abstract}
The most influential factor in the poor healing of chronic ulcers is replicative senescence of fibroblasts that are unresponsive to TGF- $\beta 1$ stimulation. Cellular replicative senescence can be induced by cultivating normal human dermal fibroblasts (HDFs) in a serum-starved medium. In addition, increasing microenviroment mechanical forces by hyaluronic acid can ameliorate the TGF- $\beta 1$ signaling of these senescent cells. One of natural resources of hyaluronic acid is bovine vitreous gel. In order to evaluate the effect of bovine-vitreous gel on replicative senescence of fibroblasts, we used various levels of bovine vitreous gel diluted in Dulbecco's modified Eagle's medium to stimulate cellular activities of serum-starved HDFs. Those cellular activities were compared to the control media, standardized hyaluronic acid, and to normal HDFs. Our results show that replicative senescence of HDFs treated with $50 \%$ bovine vitreous gel exhibited a significantly higher proliferation index, migration rate, and collagen deposition than those cultured in control media, and they displayed an equal level of cellular activity with the HDFs exposed only to standardized hyaluronic acid. We concluded that bovine vitreous gel can be used to stimulate replicative senescence of HDFs and therefore a potential candidate material to stimulate healing of chronic ulcers.
\end{abstract}

KEYWORDS bovine vitreous gel; cellular proliferation; collagen deposition; replicative senescence; HDF

\section{Introduction}

Chronic ulcers have a slow healing rate due to the predominant presence of replicative senescent fibroblasts on the ulcer-bed, which exhibit limited in: proliferation ability, migration, and extracellular matrix synthesis (Mendez et al. 1998; Harding et al. 2005; Wall et al. 2008; Brem et al. 2008). Replicative senescent cells may be present due to inefficient growth factors (GFs), which can result from low blood supply caused by capillary wall thickening (arising from diabetes induced microangiopathy) (Chia and Tang 2014), degradation of GFs by bacterial-biofilm producing proteases (McCarty et al. 2012), or trapping of GFs by fibrins of venous stasis (Trent et al. 2005). The inefficient supply of GFs correspondingly down regulates expression of the TGF- $\beta 1$ receptor on the fibroblast membranes (Cowin et al. 2001; Kim et al. 2003).

A later study carried out on human skin fibroblasts (HDFs) indicated that the improvement of fibroblast extracellular mechanical supports with hyaluronic acid (HA) can restore cellular proliferation and extracellular matrix synthesis in senescent fibroblasts. This change occurs due to cross-linking between hyaluronic acid and collagen fibrils, which can reinforce fibroblast extracellular mechanical support, change fibroblast cellular morphologies, and ultimately ameliorate the TGF- $\beta 1$ signaling pathway (Quan et al. 2013). This in vitro result is parallel with clinical findings where treatment with a hyaluronic regenerative matrix indicate significantly acceleration in healing of chronic ulcers (Motolese et al. 2013).

Mammalian vitreous gel is a special type of highly hydrated extracellular matrix composed of interwoven networks of macromolecules, such as hyaluronans and collagen fibrils. Importantly, these macromolecules also exhibit little significant quantitative variation among different mammals (Theocharis et al. 2008), although bovine vitreous gel contains higher hyaluronic acid levels than other sources, such as Streptococcus zooepidemicus or rooster comb (Shiedlin et al. 2004). Additionally, bovine vitreous gel can be obtained from bovine eye-balls, which are found readily from slaughterhouse waste. Note that bovine eye balls have never been used as biologic mate- 
rials for the healing of chronic ulcers. Here, we report the effect of bovine vitreous gel on the cellular activity of serum-starved inducing replicative senescent HDFs.

\section{Materials and methods}

The research was approved by the Ethics Committee of Biomedic Research, Faculty of Medicine, University of Gadjah Mada, Yogyakarta. HDFs of the fourth passage were isolated and cultivated from six foreskins of circumsized boys (11-13 years old) with informed consent.

\subsection{Cell cultures and conditions}

HDFs were obtained from the dermis of human foreskins by the explants technique. After disinfecting the foreskins with $10 \%$ povidone iodine and washing with phosphate buffered saline (PBS), the epidermis was cut and removed from the tissue and the dermal layer was minced into pieces 2-4 $\mathrm{mm}^{3}$ in size. These dermal tissues were then placed in a culture flask, immersed in Dulbecco's Modified Eagle's Medium (DMEM, Gibco ${ }^{\circledR}$, USA), containing $10 \%$ fetal bovine serum (FBS, Gibco ${ }^{\circledR}$, USA) and $1 \%$ penicillin/streptomycin (Gibco ${ }^{\circledR}$, USA) at $37^{\circ} \mathrm{C}$ continuously until the tissues adhered to the bottom of the flask. The medium then was changed every $72 \mathrm{~h}$ until the $60 \%$ of the HDF-outgrowth could be observed under an inverted microscope. The cells then were harvested and sub-cultured until fourth passage.

\subsection{Serum-starved HDFs}

Artificially-senescent HDFs from each foreskin were driven by culturing fourth passage of HDFs based on a previously reported method (Alcorta et al. 1996). Briefly, $200 \mu \mathrm{L}$ of HDFs suspension of $5 \times 10^{3}$ cells/mL growth medium were seeded into each well of 96 well plates. After incubation for $24 \mathrm{~h}$ at $37^{\circ} \mathrm{C}$ in a humidified atmosphere with $5 \% \mathrm{CO}_{2}$, the medium was removed, rinsed with PBS, and cultured in DMEM containing 0.1\% FBS. Furthermore, cells were cultured in triplicate-pair in growth medium and used as standard HDFs. All HDFs cultures were incubated for $72 \mathrm{~h}$ at $37^{\circ} \mathrm{C}$ with $5 \% \mathrm{CO}_{2}$.

\subsection{Preparation of bovine vitreous gel}

Six fresh bovine eye-balls were collected from healthy cattle from a licensed slaughterhouse. The eyeballs were retrieved immediately after slaughter, and they were individually placed in tubes containing PBS in an ice box. Each eyeball was then washed with $10 \%$ povidone iodine and then three times with normal saline. The vitreous gel was aspirated by the aseptic technique, transferred into a sterile tube, and centrifuged with $200 \mathrm{~g}$ for $10 \mathrm{~min}$. The supernatant was then isolated and stored at $4^{\circ} \mathrm{C}$ for later use.

\subsection{Experiments}

All experimental steps are summarized in Figure 1.

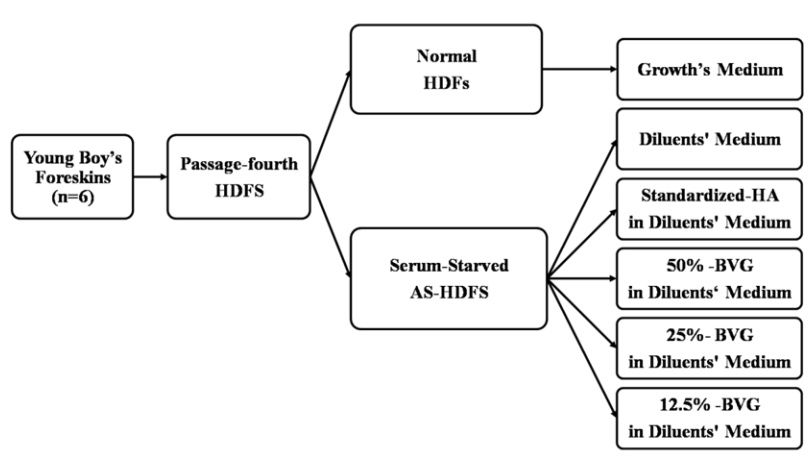

FIGURE 1 Summary of the experimental research design. HDFS = human dermal fibroblasts; AS-HDFS = artificially-senescent HDFs; BVG = bovine's vitreous gel; $\mathrm{HA}=$ hyaluronic acid.

After aspirating the medium, the cells were washed three times with PBS. Subsequently, a $200 \mu \mathrm{L}$ mixture of growth medium, dilution medium, $0.0625 \mathrm{mg} / \mathrm{mL} \mathrm{HA} \mathrm{(}{ }^{\circledR}$ Derma-Kirei-Japan) in dilution medium, and various levels of BVG in dilution medium were added to targeted cells as illustrated in Figure 1. The cells were incubated for $72 \mathrm{~h}$ at $37^{\circ} \mathrm{C}$ in a humidified atmosphere with $5 \% \mathrm{CO}_{2}$. All procedures were replicated three times.

\subsection{Measurements}

\subsubsection{Proliferation index}

Cellular viabilities were measured by using a standardized MTT (3-(4,5-dimethylthiazol-2-yl)-2,5-diphenyltetrazolium bromide, purchased from MP Biomedicals, France) assay, and the optical density of formed formazan blue was measured at a wavelength of $570 \mathrm{~nm}$ using a spectrophotometer.

The proliferation index of normalHDFs was assumed to be $100 \%$ and the proliferation index of various treated groups was determined as follows:

$$
\begin{aligned}
& \text { Proliferation index of treated group } \\
& =\frac{\text { Treated group’s cellular viabilities }}{\text { Paired normal HDF's cellular viabilities }} \times 100 \%
\end{aligned}
$$

\subsubsection{Collagen deposition}

The quantity of collagen was measured using an insoluble collagen Sirius Red assay (YERCAN and KUTAY 1999). The Sirius Red bound to insoluble collagen was dissolved using $200 \mu \mathrm{L}$ of $0.5 \mathrm{~N} \mathrm{NaOH}$ and the optical density was detected at $570 \mathrm{~nm}$ using a spectrophotometer. The collagen deposition of normal HDFs was taken to be $100 \%$ and collagen deposition in various treated groups was determined using the following formula: 


\author{
Collagen deposition of treated group \\ $=\frac{\text { Insoluble collagen of treated group }}{\text { Insolution collagen of paired normal HDFs }} \times 100 \%$
}

\subsubsection{Cellular migration assay}

A cellular migration assay was performed based on a method that was introduced previously by Yarrow et al. (2004). Cellular migration was computed based on the method of Liang et al. (2007) with slight modification. Briefly, after serum starvation all wells were linearly scratched with the blunt tip of a $32 \mathrm{G}$ sterile needle through the center of the well bottom. After appropriate washing with PBS for removing scratched cells, the cells were then cultivated in the experimental medium for $72 \mathrm{~h}$. All cells on linearly scratched areas were considered as migrated cells (Figure 2). The cells then were stained with Meyer's haematoxylin and microscopic photo images were taken in JPG format using a Moticam 350 (China) camera. By using Adobe Photoshop, it was possible to identity the blue pixels of fibroblasts along the scratch line and white pixels corresponding to empty space. Cell migration capacity was determined using the following equation:

\author{
Migration \\ $=\frac{\text { Blue color pixel fibroblast along scratch line }}{\text { Total pixels along scratch line }} \times 100 \%$
}

\subsubsection{Statistics}

All data are presented as mean \pm standard error. We used analysis of variance (ANOVA) followed by Least Significant Difference (LSD) to analyze the migration rate data and Friedman test followed by Wilcoxon as a post-hoc test to analyze the proliferation index and collagen deposition data. The level of statistical significance was at $p<0.05$.

\section{Results}

Our experiments showed that serum-starved medium causes supression of HDF activity, specifically the cell proliferation index was reduced by $48.52 \%$, collagen deposition capacity was reduced by $60.50 \%$, and cellular migration capacity was reduced by $73.25 \%$. Treatment with hyaluronic acid indicated that those activities can be stimulated up to $59.83 \%, 82.99 \%$, and $96.80 \%$, respectively $(p<0.05)$. Treatment with $50 \%$ BVG indicated that pro-

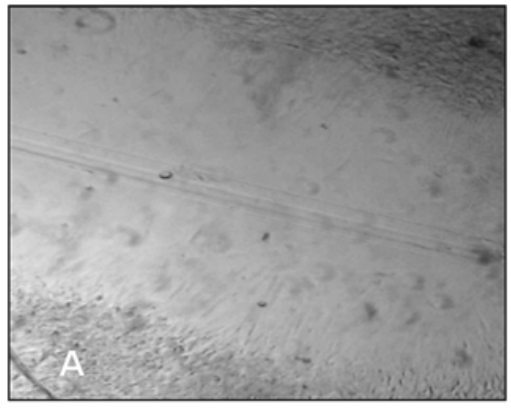

(a)

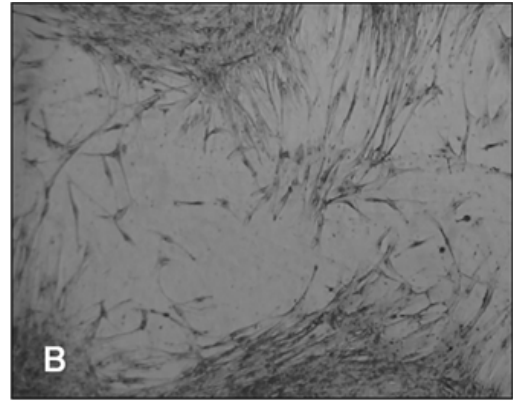

(b)

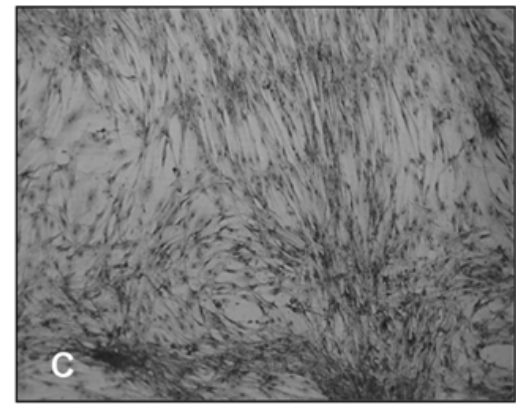

(c)

FIGURE 2 Scratch assay: (a) immediately after scratching - no staining; (b) $24 \mathrm{~h}$ after scratching - hematoxylin eosin; (c) $48 \mathrm{~h}$ after scratching - hematoxylin eosin.

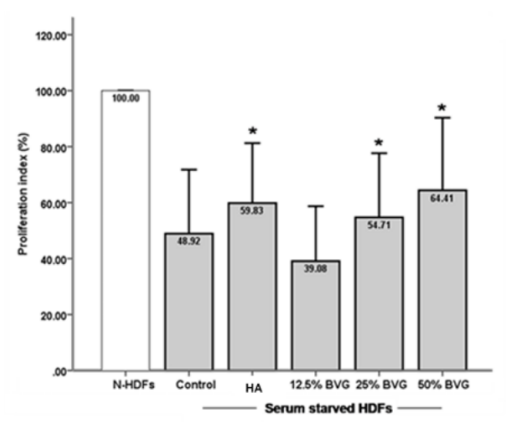

(a)

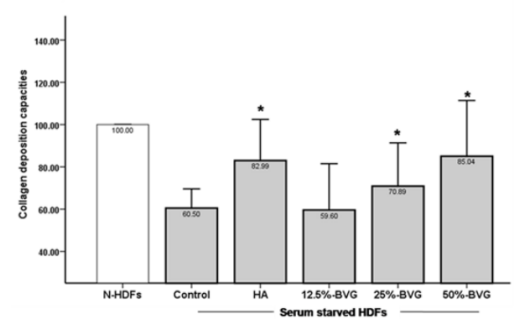

(b)

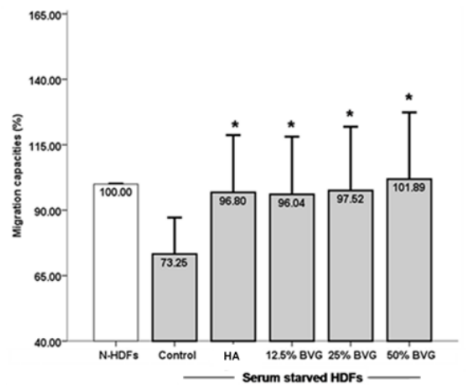

(c)

FIGURE 3 The activities of serum-starved human dermal fibroblast under BVG-medium: (a) proliferation index among various treated groups; (b) collagen deposition capacity among various treated groups; (c) migration capacity among various treated groups. ${ }^{*}=p<0.05$ compared with control; N-HDFs = normal human dermal fibroblast; BVG = bovine's vitreous gel; HA = hyaluronic acid. 


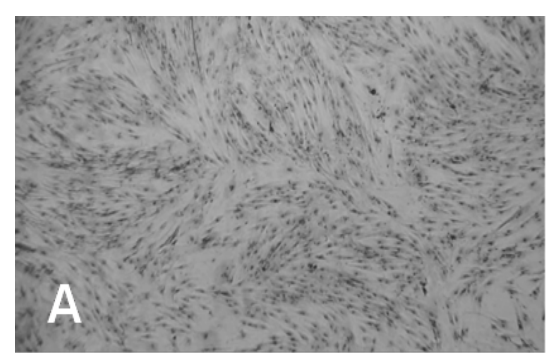

(a)

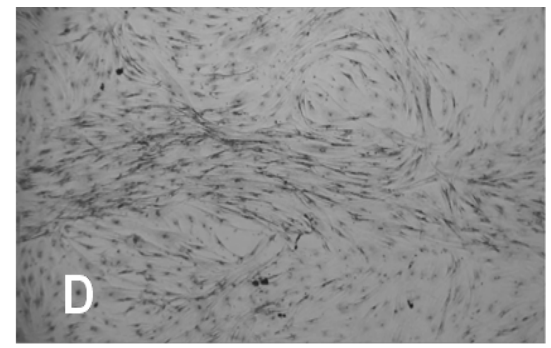

(d)

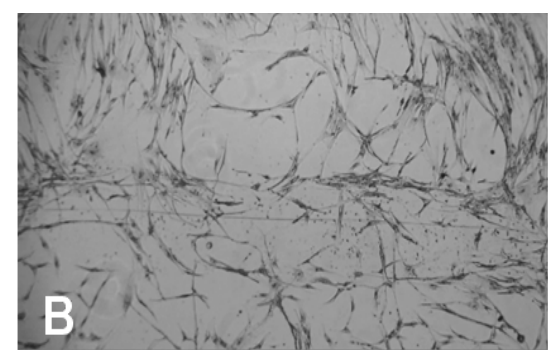

(b)

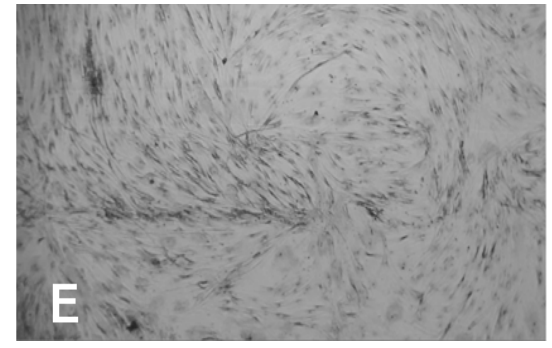

(e)

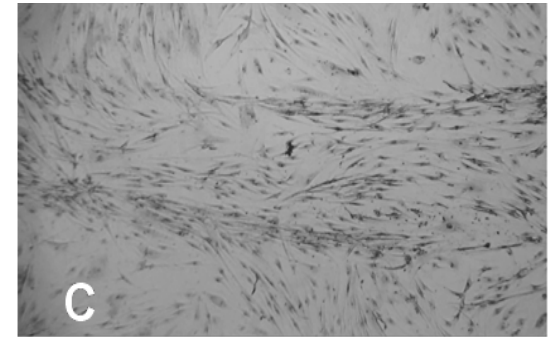

(c)

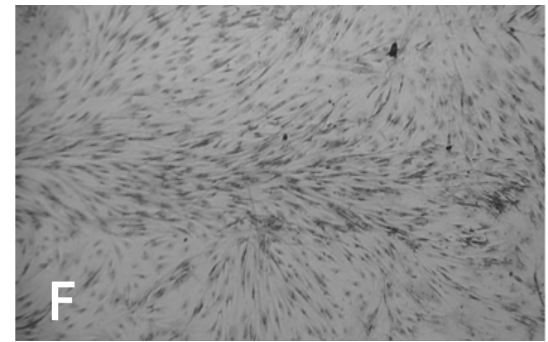

(f)

FIGURE 4 Scratch assay of $72 \mathrm{~h}$ incubation: (a) normal HDFs; (b) serum-starved HDFs in control; (c) serum-starved HDFs in HA; (d) serumstarved HDFs in 12.5\% BVG; (e) serum-starved HDFs in 25\% BVG; (f) serum-starved HDFs in 50\% BVG.

liferation index was raised to $64.41 \%$, Collagen deposition capacity was to $85.04 \%$, and migration capacity was to $101.89 \%$ significantly ( $p<0.05 \%)$. These results are given in Figure 3 and Figure 4.

\section{Discussion}

Previous research has indicated that fibroblasts cultivated from chronic ulcer beds may proliferate slowly (Almqvist et al. 2009). In order to obtain large number of fibroblasts, dermal fibroblasts from young human skin then were subsequently stimulated toward replicative senescence by various methods. The simplest method utilized was cultivation of monolayer normal fibroblasts in a serum-starved medium for 72 h (Alcorta et al. 1996). In this condition, serum restriction was a way to create fibroblasts environment with limit the GF supply, in an attempt to mimic the extracellular fluid environment in chronic ulcers.

The characteristic of replicative senescence fibroblasts is decreasing ability of those cells in: proliferation, migration, and synthesize extracellular matrix of collagen. In our experiment, compared to normal HDFs, serumstarved fibroblasts exhibited a decreasing of proliferation index, collagen deposition capacity, and migration capacity. These findings are not only indicated characteristic of senescence fibroblasts, but they also displayed the characteristics of dermal fibroblasts isolated from the margins of venous chronic ulcers (Stanley and Osler 2001; Harding et al. 2005). Previous experiments have also shown that cultivation of HDFs in a low-serum medium can stimulate replicative senescence in HDFs through decreasing expression of the membrane-type II receptor of TGF- $\beta 1$ (Kim et al. 2003).
Our experiments indicate that adding BVG in a lowserum medium can significantly reactivate serum-starved HDFs with regard to their proliferation index, collagen deposition capacity, and migration capacity $(p<0.05)$, especially for BVG levels of $25 \%$ and $50 \%$ (Figure 3 ). Improvement of migration capacities of serum-starved HDFs in $25 \%$ and $50 \%$ BVG could be observed $72 \mathrm{~h}$ of incubation (Figure 3 and Figure 4).

Importantly, these findings show that BVG has the ability to regenerate the function of serum-starved HDFs. This regeneration occurs because BVG contains high levels of HA (Shiedlin et al. 2004). Indeed, previous a study showed that HA could enhance fibroblast extracellular structural mechanical supports, change fibroblast cellular morphologies, re-expose of hidden TGF- $\beta 1$ receptor and ameliorate the TGF- $\beta 1$ signaling pathway in procollagen synthesis (Quan et al. 2013). As well known previously, binding TGF- $\beta 1$ on its receptor may stimulate procollagen synthesis through smad 3 activation. Secreted pro-collagen is then suffering various events such as hydroxylation, glycocylation, and triple helix formation to perform deposited collagen. Deposition collagen on extracellular does not only affect extracellular matrix composition for media for fibroblast migration, but also affects extracellular mechanical force to change the shape of fibroblast become more stretched, TGF- $\beta 1$ receptor is then more exposed. In this condition, mechanical stretching of fibroblasts more ameliorates the TGF- $\beta 1$ signaling pathway, and it also stimulates these cells to produce growth factors and metalloproteases tissue inhibitors as a preventative agent for metalloproteinase (Eckes et al. 2006). Reducing activity of metalloproteinase may increase collagen deposition due to lowering collagen degradation. Col- 
lagen deposition is the result of new collagen synthesis, minus any collagen degradation due to metalloproteinase activities.

There was not any significant difference between serum-starved HDFs with HA exposure and with 50\% BVG, with regard to proliferation index, collagen deposition capacity, and migration capacity $(p>0.05)$, although the result with BVG was slightly better than the one with HA. The slight improvement may be due to the fact that BVG contains not only HA but also collagen and GFs (Courty et al. 1986).

One of our study limitations was the absence of data for the detection of HDF replicative senescence for those cells cultured in serum-starved medium. Replicative senescence of HDFs can be detected by senescenceassociated galactosidase staining, and this is a method commonly used by many researchers. By not carrying out senescence-associated galactosidase staining in this study, replicative senescence of targeted cells could not be confirmed with certainty. The study also did not confirm TGF$\beta 1$ signaling in treated cells by signaling protocol, and therefore the improvements observed for those cells are uncertain due to the re-expression of TGF- $\beta 1$ receptors on fibroblast membranes.

\section{Conclusions}

It can be concluded that, when used as hydrated extracellular matrix, BVG can reactivate serum-starved HDFs as effectively as hyaluronic acid. This research may therefore serve as a starting point for futurefor animal studies and clinical studies, so that clinical application of BVG may ultimately be used as a treatment for chronic ulcers.

\section{Acknowledgments}

This research was financially supported by the 2015 Dana Masyarakat of the Faculty of Medicine, Universitas Gadjah Mada, Yogyakarta.

\section{Authors' contributions}

All of the authors contributed to the design, conducting of experiments, analysis of the results, and the writing of the manuscript. YWW was also responsible for correcting the manuscript according to the reviewers' suggestions.

\section{Competing interests}

All of the authors declare that there is no significant competing financial, professional, and personal interest that might have influenced this manuscript.

\section{References}

Alcorta D, Xiong Y, Phelps D, Hannon G, Beach D, Barrett J. 1996. Involvement of the cyclin-dependent ki- nase inhibitor p16 (INK4a) in replicative senescence of normal human fibroblasts. Proc Natl Acad Sci USA 93(24):13742-13747. doi:10.1073/pnas.93.24.13742.

Almqvist S, Werthén M, Johansson A, Törnqvist J, Agren MS, Thomsen P. 2009. Evaluation of a nearsenescent human dermal fibroblast cell line and effect of amelogenin. Br J Dermatol. 160(6):1163-1171. doi:10.1111/j.1365-2133.2009.09071.x.

Brem H, Golinko MS, Stojadinovic O, Kodra A, Diegelmann RF, Vukelic S, Entero H, Coppock DL, TomicCanic M. 2008. Primary cultured fibroblasts derived from patients with chronic wounds: a methodology to produce human cell lines and test putative growth factor therapy such as GMCSF. J Transl Med. 6:75. doi:10.1186/1479-5876-6-75.

Chia HY, Tang MBY. 2014. Chronic leg ulcers in adult patients with rheumatological diseases - a 7-year retrospective review. Int Wound J. 11(6):601-604. doi:10.1111/iwj.12012.

Courty J, Chevallier B, Moenner M, Loret C, Lagente O, Bohlen P, Courtois Y, Barritault D. 1986. Evidence for FGF-like growth factor in adult bovine retina: Analogies with EDGF I. Biochem Biophys Res Commun. 136(1):102-108. doi:10.1016/0006291X(86)90882-X.

Cowin AJ, Hatzirodos N, Holding CA, Dunaiski V, Harries RH, Rayner TE, Fitridge R, Cooter RD, Schultz GS, Belford DA. 2001. Effect of healing on the expression of transforming growth factor beta(s) and their receptors in chronic venous leg ulcers. J Invest Dermatol. 117(5):1282-1289. doi:10.1046/j.0022202x.2001.01501.x.

Eckes B, Zweers MC, Zhang ZG, Hallinger R, Mauch C, Aumailley M, Krieg T. 2006. Mechanical tension and integrin alpha 2 beta 1 regulate fibroblast functions. J Investig Dermatol Symp Proc. 11(1):66-72.

Harding KG, Moore K, Phillips TJ. 2005. Wound chronicity and fibroblast senescence-implications for treatment. Int Wound J. 2(4):364-368. doi:10.1111/j.1742-4801.2005.00149.x.

Kim BC, Kim HT, Park SH, Cha JS, Yufit T, Kim SJ, Falanga V. 2003. Fibroblasts from chronic wounds show altered TGF-beta-signaling and decreased TGFbeta Type II receptor expression. J Cell Physiol. 195(3):331-336. doi:10.1002/jcp.10301.

Liang CC, Park AY, Guan JL. 2007. In vitro scratch assay: a convenient and inexpensive method for analysis of cell migration in vitro. Nat Protoc. 2(2):329-333. doi:10.1038/nprot.2007.30.

McCarty SM, Cochrane CA, Clegg PD, Percival SL. 2012. The role of endogenous and exogenous enzymes in chronic wounds: a focus on the implications of aberrant levels of both host and bacterial proteases in wound healing. Wound Repair Regen. 20(2):125-136. doi:10.1111/j.1524-475X.2012.00763.x. 
Mendez MV, Stanley A, Park HY, Shon K, Phillips T, Menzoian JO. 1998. Fibroblasts cultured from venous ulcers display cellular characteristics of senescence. J Vasc Surg. 28(5):876-883. doi:10.1016/s07415214(98)70064-3.

Motolese A, Vignati F, Brambilla R, Cerati M, Passi A. 2013. Interaction between a regenerative matrix and wound bed in nonhealing ulcers: results with 16 cases. Biomed Res Int. 2013:849321. doi:10.1155/2013/849321.

Quan T, Wang F, Shao Y, Rittié L, Xia W, Orringer JS, Voorhees JJ, Fisher GJ. 2013. Enhancing structural support of the dermal microenvironment activates fibroblasts, endothelial cells, and keratinocytes in aged human skin in vivo. J Invest Dermatol. 133(3):658667. doi:10.1038/jid.2012.364.

Shiedlin A, Bigelow R, Christopher W, Arbabi S, Yang L, Maier RV, Wainwright N, Childs A, Miller RJ. 2004. Evaluation of hyaluronan from different sources: Streptococcus zooepidemicus, rooster comb, bovine vitreous, and human umbilical cord. Biomacromolecules 5(6):2122-2127. doi:10.1021/bm0498427.

Stanley A, Osler T. 2001. Senescence and the healing rates of venous ulcers. J Vasc Surg. 33(6):1206-1211. doi:10.1067/mva.2001.115379.

Theocharis DA, Skandalis SS, Noulas AV, Papageorgakopoulou N, Theocharis AD, Karamanos NK. 2008. Hyaluronan and chondroitin sulfate proteoglycans in the supramolecular organization of the mammalian vitreous body. Connect Tissue Res. 49(3):124-128. doi:10.1080/03008200802148496.

Trent JT, Falabella A, Eaglstein WH, Kirsner RS. 2005. Venous ulcers: pathophysiology and treatment options. Ostomy Wound Manage. 51(5):38-54; quiz 5556.

Wall IB, Moseley R, Baird DM, Kipling D, Giles P, Laffafian I, Price PE, Thomas DW, Stephens P. 2008. Fibroblast dysfunction is a key factor in the non-healing of chronic venous leg ulcers. J Invest Dermatol. 128(10):2526-2540. doi:10.1038/jid.2008.114.

Yarrow JC, Perlman ZE, Westwood NJ, Mitchison TJ. 2004. A high-throughput cell migration assay using scratch wound healing, a comparison of imagebased readout methods. BMC Biotechnol. 4:21. doi:10.1186/1472-6750-4-21.

YERCAN H, KUTAY FZ. 1999. Quantification of total collagen in rabbit tendon by the Sirius Red Method. Turk J Med Sci. 29:7-9. 